\title{
Reviewer acknowledgement Bot. Mar. volume 50 (2007)
}

The editors and the publishers wish to thank the following colleagues for their kind assistance in acting as referees for the journal this year:

\author{
Julio Afonso-Carrillo \\ Ana Amorim \\ Charles D. Amsler \\ Robert A. Andersen \\ Put O. Ang \\ Betsy Arnold \\ Julio Arrontes \\ Knud Erik Bach Knudsen \\ David Balata \\ Elena Balestri \\ John W. Blunt \\ John J. Bolton \\ Fernando G. Brun \\ Alejandro $\mathrm{H}$. Buschmann \\ Jinx Campbell \\ Just Cebrian \\ Susana Coelho \\ Zvi Cohen \\ Clinton J. Dawes \\ Lou Francis De Filippis \\ Valery M. Dembitsky \\ Thiruvengadam Devaki \\ John D. Dodge \\ Christian Drapeau \\ Matthew J. Dring \\ Lars Edler \\ Bente Edvardsen \\ Wenche Eikrem \\ Malte Elbrächter \\ Aschwin H. Engelen \\ Sophia Fox \\ Giovanni Furnari \\ James B. Gloer \\ Iván Gómez \\ Marie-Laure Guillemin \\ Gustaaf M. Hallegraeff \\ Richard T. Hanlin \\ John L. Harwood \\ Claire Hellio
}

Iris Hendriks

Helmut Hillebrand

Xiao Yan Hu

John M. Huisman

Paul Jensen

Jacob John

John Johnson

Joanna M. Jones (Kain)

Gwang Hoon Kim

Jan Kohlmeyer

Vladimir Kostylev

Gerald T. Kraft

Hartmut Laatsch

Samuel Laney

Michael I. Latz

Yves Le Gal

Jeannie-Marie LeRoi

Amy Leventer

Nina Lundholm

Jesús Mercado

Kathy Ann Miller

J. David Miller

Richard Moe

Steven N. Murray

Nelson Navarro

Carolanne Nelson

F. Xavier Niell

James Nienow

Vincent Ooi

Perfecto Paseiro-Losada

Renato C. Pereira

Bernardo A. Perez da Gama

Naomi Phillips

Antonio Pollio

Simeon Popov

Peter Proksch

Willem F. Prud'homme van Reine

Maria L. Quartino

Thorsten Reusch
Fabio Rindi

André Rochon

Koen Sabbe

Peter Saenger

Ronelie Chato Salvador

Martha Sansón

Diana Sarno

Britta Schaffelke

Scott Schatz

John Paul Schmit

Craig W. Schneider

Abel Sentíes Granados

Donatella Serio

Ester Serrão

Alison Sherwood

Paul C. Silva

Martin J. Stillman

Wendy A. Stirk

Ana Maria Suárez

Michael J. Sullivan

Mirta Teichberg

Jorge Terrados

Edward C. Theriot

Gavino C. Trono

Wendy Untereiner

Anatolii I. Usov

Robert Vadas

Joseph P. Valentine

Kathryn L. Van Alstyne

Johannes Van Staden

Brigitta I. van Tussenbroek

Juan Vergara

Marc Verlaque

Maria C. Villac

Annemiek Vink

Bin-Gui Wang

Martin Wilkinson

Thebtaranonth Yodhathai

Adriana Zingone 